\title{
Energy Based Clutter Filtering for Vector Flow Imaging
}

Villagómez Hoyos, Carlos Armando; Jensen, Jonas; Ewertsen, Caroline; Lindskov Hansen, Kristoffer; Nielsen, Michael Bachmann; Jensen, Jørgen Arendt

\section{Published in:}

Proceedings of IEEE International Ultrasonics Symposium

\section{Publication date:}

2017

Document Version

Peer reviewed version

Link back to DTU Orbit

Citation (APA):

Villagómez Hoyos, C. A., Jensen, J., Ewertsen, C., Lindskov Hansen, K., Nielsen, M. B., \& Jensen, J. A. (2017). Energy Based Clutter Filtering for Vector Flow Imaging. In Proceedings of IEEE International Ultrasonics Symposium IEEE.

\section{General rights}

Copyright and moral rights for the publications made accessible in the public portal are retained by the authors and/or other copyright owners and it is a condition of accessing publications that users recognise and abide by the legal requirements associated with these rights.

- Users may download and print one copy of any publication from the public portal for the purpose of private study or research.

- You may not further distribute the material or use it for any profit-making activity or commercial gain

- You may freely distribute the URL identifying the publication in the public portal

If you believe that this document breaches copyright please contact us providing details, and we will remove access to the work immediately and investigate your claim. 


\title{
Energy Based Clutter Filtering for Vector Flow Imaging
}

\author{
Carlos A. Villagómez-Hoyos, Jonas Jensen, Caroline Ewertsen, Kristoffer L. Hansen, \\ Michael B. Nielsen and Jørgen A. Jensen \\ Center for Fast Ultrasound Imaging, Department of Electrical Engineering, \\ Technical University of Denmark, DK-2800 Lyngby, Denmark
}

\begin{abstract}
To obtain accurate blood flow velocity estimates it is important to remove the clutter signal originating from tissue. Conventionally, the clutter signal has been separated from the blood signal based on the difference of their spectral frequencies. However, this approach is not enough for obtaining vector flow measurements, since the spectra overlaps at high beam-to-flow angles. In this work a distinct approach is proposed, where the energy of the velocity spectrum is used to differentiate among the two signals. The energy based method is applied by limiting the amplitude of the velocity spectrum function to a predetermined threshold. The effect of the clutter filtering is evaluated on a plane wave (PW) scan sequence in combination with transverse oscillation (TO) and directional beamforming (DB) for velocity estimation. The performance of the filter is assessed by comparison of the velocity estimates of the proposed filter against a conventional moving average clutter filter. The effect of tissue motion is investigated using a Field II simulation of a straight vessel with moving wall, while the direct effect of the filter on the velocity estimates is evaluated on a CFD model of a carotid bifurcation with a fixed vessel wall. The results show that the proposed filter outperformed the moving average during moving vessel wall conditions, where standard deviations from the velocity magnitudes and angles were kept consistently below $6 \%$ and $6^{\circ}$ compared to $63 \%$ and $48^{\circ}$ on the moving average filter. The results on the CFD showed that on non-moving conditions the velocity estimates had minor statistical differences with errors on the magnitude of $\mathbf{- 7 . 9 5} \pm \mathbf{1 0 . 1 \%}$ and angles of $0.15 \pm 6.65$ for the proposed filter compared to $\mathbf{- 5 . 8 3} \pm \mathbf{9 . 0 8} \%$ and $\mathbf{- 0 . 1 2} \pm \mathbf{4 . 4 8}$.
\end{abstract}

\section{INTRODUCTION}

To get accurate velocity estimates in vector flow imaging (VFI), it is essential to separate the blood signal from the clutter signals originating from the tissue, especially when tissue motion is present. Motion presents an even bigger challenge for vector flow estimators than for conventional color flow mapping (CFM). The reason being that at high beam-to-flow angles the overlap between the frequency spectra from tissue and blood is increased.

Over the past years, the design principle for clutter removal has remain basically the same. The clutter signal has been separated from the blood signal based on the difference of their spectral frequencies. This design is not convenient for vector flow due to the larger overlap in the frequency spectra at high beam-to-flow angles, requiring filter with steep cutoff and adaptive behaviour. To overcome this limitation, it is proposed to use the energy level of the spectrum instead of the frequency as a discriminator. The use of the energy or amplitude characteristic as a discriminator is not new, and has previously been indirectly incorporated in other tissue echocanceling methodologies such as eigen-vectors [1] or singular value decomposition [2]. The difference relies on that these methods uses a basis transformation to account for the energy content. However, the generated transform basis is unique, and it is challenging to decide how many components from the new basis to remove. These methods sometimes require an additional Fourier transform of the new basis for further decision making [3].

This work presents an energy based filter scheme to better suppress the clutter signal originating from a moving clutter signal. The filter operates on the Fourier basis, to facilitate the analysis of the signals. The filter theory, which describes the principle of operating on the energy content instead of frequency, is explained in Section II. The methodologies of velocity estimation, simulations of a straight vessel with moving wall and CFD model of a carotid bifurcation are explained in Section III. The results of comparing the velocity estimates of the proposed filter against a conventional moving average clutter filter are presented in Section IV. This is followed by a discussion of the influence of the threshold selection and a conclusion.

\section{THEORY}

The signal from moving tissue and blood can be represented by a 1-D statistical Gaussian distribution, according to the model developed by Torp et al [4]. The velocity spectrum, thus, is expressed as

$$
\begin{aligned}
& H(\omega)=G_{\text {tissue }}(\omega)+G_{\text {blood }}(\omega), \\
& \text { where } \\
& \qquad G(\omega)=R(0) \sqrt{2 \pi} \sigma \exp ^{-\frac{1}{2}\left(\omega-\omega_{d}\right)^{2} \sigma^{2} .}
\end{aligned}
$$

The mean frequency is given by the Doppler frequency $\omega_{d}$, the bandwidth by $\sigma$; which is related to the transit-time of the scatterers (see [5] for definition), and total energy of the signal expressed as the auto-correlation function at zero lag $R(0)$ of each signal.

The energy based approach is applied by limiting the amplitude of the velocity spectrum function $H(\omega)$ to a predetermined threshold $A_{c}$, as illustrated in Fig. 1. The threshold should 
ideally be selected to be just above $G_{b l o o d}$ peak value. The tissue signal removal process relies on Parseval's theorem, which states that the total energy of a signal and the sum of the energy components of the signal's Fourier transform are equal. Therefore, by limiting $H(\omega)$ to $A_{c}$, the energy content of the tissue signal is reduced while maintaining the blood signal.

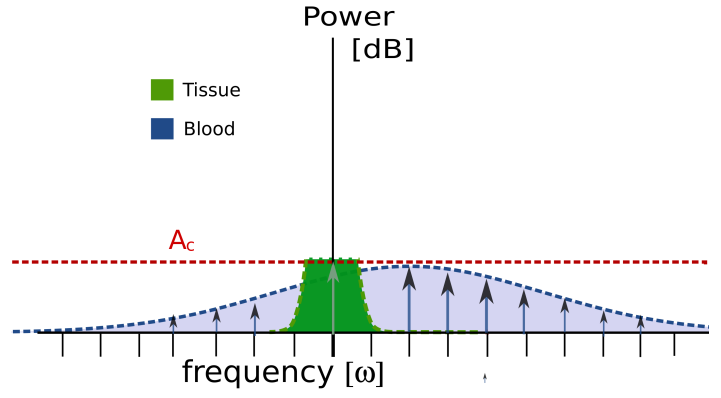

Fig. 1. Fourier spectra of the tissue (green) and blood (blue) signal. The tissue spectra has been limited by the amplitude cut-off (red), which effectively reduces the energy level of the tissue signal.

For the blood signal to overpower the tissue signal, the area under the curve for the blood signal must be larger than the one belonging to the tissue. A condition for this could be that a broader blood bandwidth is present $\left(\sigma_{\text {blood }}>\sigma_{\text {tissue }}\right)$. This is fortunate, since blood signals carry broader bandwidths due to the transit-time effects originated by the movement of the blood scatterers. However, slow moving blood flow with reduced $\sigma_{\text {blood }}$ or large tissue signal sidelobes due to spectral leakage or insufficient sampling, could preclude the condition to be met. The inverse Fourier transform of $H(\omega)$ will still contain a combination of tissue and blood signals, but with the blood signal overpowering the tissue's.

\section{METHODS}

A plane wave (PW) scan sequence is performed using a linear array transducer $(0.2 \mathrm{~mm}$ pitch , 192 elements, $38 \mathrm{~mm}$ elevation focus, $6 \mathrm{~mm}$ height), A duplex sequence consisting of both flow and B-mode emissions is employed (4.1 MHz center frequency, $10 \mathrm{kHz}$ PRF, plane waves at $-15^{\circ}, 0^{\circ}, 15^{\circ}$ for flow sequence), details on the B-mode sequence at [7]. The evaluated moving average has a packet length of 32 and the energy based uses a packet length of 128 .

The velocity estimation is based on a combination of transverse oscillation (TO) and directional beamforming (DB) and is carried out as described in [7]. The velocity estimation is a two-step procedure; 1) the TO method is used to estimate an initial flow angle $\theta$, which is calculated from the axial and lateral velocities $v_{z}$ and $v_{x}$, respectively. 2) three DB lines are focused around $\theta$ at each estimation point to find a refined angle $\hat{\theta}$ using the largest normalized cross-correlation estimate. The velocity magnitude is obtained along the direction $\hat{\theta}$ using a cross-correlation estimator.

\section{A. Straight vessel}

Vessels with a diameter of $5 \mathrm{~mm}$ centred at $30 \mathrm{~mm}$ are simulated with the PW sequence and Field II Pro [8]. Blood scatterers are moved using a parabolic profile with a peak velocity of $0.25 \mathrm{~m} / \mathrm{s}$. Additionally to the blood scatterers, a concentric expanding vessel wall is simulated and moved at velocities of $0,1.25,2.5$, and $3.75 \mathrm{~mm} / \mathrm{s}$. The vessel wall scatterers mean amplitudes are set $10 \mathrm{~dB}$ above the tissue signal mean amplitude, while the blood flow is set $40 \mathrm{~dB}$ below. The vessels are simulated with beam-to-flow angles of $60^{\circ}$ and $90^{\circ}$.

\section{B. CFD Bifurcation model}

The carotid bifurcation CFD dataset from Swillens et al. work is used [9], in which the carotid bifurcation geometry is generated from of a healthy volunteer and an artificially eccentric plaque is inserted in the interna. The CFD-package Fluent with an imposed inlet velocity profile obtained from a Doppler measurement of the same volunteer are used to simulate the velocities. Rigid walls are assumed in the CFD model, and no vessel wall or tissue movement is present.

The CFD model is coupled to Field II using the CFD-US simulation framework also openly available from Swillens' website (@BioMMeda).
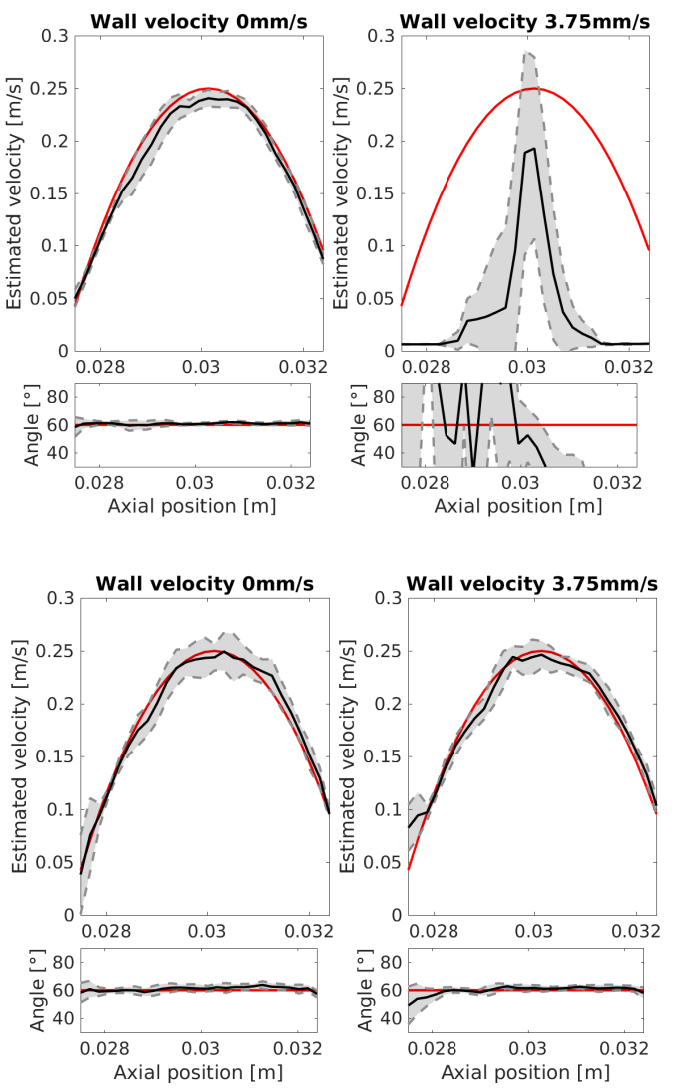

Fig. 2. Estimated velocity profiles at the $60^{\circ}$ beam-to-flow angle vessel for the moving average filter (top),the energy based filter (bottom) for different wall velocities. The expected profile for the velocity magnitude and angle are shown in red. 


\section{In vivo measurement}

A 27 year old healthy volunteer participated after informed consent, and the study is approved by the National Committee on Biomedical Research Ethics (Protocol No. H-1-2014-fsp072). A linear array with similar characteristics as the on used in the simulations is connected to the SARUS scanner [6], and element data are acquired and then beamformed in a computing cluster. The carotid bulb is scanned with the best possible view for observing both the externa and the interna of the bifurcation.

\section{RESUlts}

The results from the simulated vessel with a $60^{\circ}$ beam-toflow are shown in Fig. 2 for the moving average filter (top) and the energy based filter (bottom). The results to the left are the velocities estimates in the absence of motion, where good biases of $3.55 \%$ and $0.15 \%$ with good standard deviations of $3.54 \%$ and $5.36 \%$ are found for both the moving average and the energy based filter, respectively. However, the increased presence of vessel wall movement degrades the velocity estimates generating an increase of bias up to $-63.8 \%$ for the $3.75 \mathrm{~mm} / \mathrm{s}$ case, when using the moving average filter. The areas closer to the vessel boundaries are specially affected, since they present lower velocities. Conversely, the energy based clutter filtering shown on Fig. 2 (bottom) presents a constant behaviour independently of the vessel wall movement. This can also be observed in Table I, where the results for all the straight vessel simulations are summarized. Notably, the results from the $90^{\circ}$ vessel shows a systematic under estimation of the velocity but maintains an independent behaviour in respect to the tissue movement. The under estimation could be attributed to the selection of the windowing during the Fourier transform and should be further investigated.

Table I

MEAN BIAS AND SD FOR SIMULATED STRAIGHT VESSELS

\begin{tabular}{|c|c|c|c|c|}
\hline \multicolumn{5}{|c|}{$60[\mathrm{deg}]$} \\
\hline & \multicolumn{2}{|c|}{ Moving average } & \multicolumn{2}{|c|}{ Energy based } \\
\hline $\mathrm{mm} / \mathrm{s}$ & Velocity [\%] & Angle [deg] & Velocity [\%] & Angle [deg] \\
\hline 0 & $-3.55 \pm 3.64$ & $0.96 \pm 1.58$ & $0.15 \pm 5.36$ & $1.31 \pm 2.51$ \\
\hline 1.25 & $-50.3 \pm 11.0$ & $-10.4 \pm 53.2$ & $-0.08 \pm 4.66$ & $1.29 \pm 2.29$ \\
\hline 2.5 & $-59.7 \pm 11.2$ & $-5.3 \pm 44.8$ & $-0.11 \pm 4.45$ & $1.25 \pm 2.30$ \\
\hline 3.75 & $-63.8 \pm 13.3$ & $-7.0 \pm 48.1$ & $-0.52 \pm 4.10$ & $0.91 \pm 2.19$ \\
\hline \multicolumn{5}{|c|}{$90[\mathrm{deg}]$} \\
\hline $\mathrm{mm} / \mathrm{s}$ & Velocity [\%] & Angle [deg] & Velocity [\%] & Angle [deg] \\
\hline 0 & $-10.4 \pm 2.88$ & $-0.11 \pm 0.60$ & $-32.0 \pm 5.28$ & $-0.28 \pm 1.52$ \\
\hline 1.25 & $-31.3 \pm 4.25$ & $-1.30 \pm 7.37$ & $-33.8 \pm 5.38$ & $-0.514 \pm 1.26$ \\
\hline 2.5 & $-41.4 \pm 4.58$ & $-4.45 \pm 11.6$ & $-35.3 \pm 5.70$ & $-0.13 \pm 1.90$ \\
\hline 3.75 & $-47.2 \pm 5.05$ & $1.69 \pm 10.4$ & $-35.9 \pm 5.11$ & $-2.20 \pm 5.87$ \\
\hline
\end{tabular}

The error in the estimated velocities during the peak systole from the moving average filter $\left(V_{m a}\right)$ and the energy based filter $\left(V_{\text {ergy }}\right)$, when applied to the CFD bifurcation model simulation, are shown in Fig. 3. The graphs show the errors for the estimated velocity magnitudes (left) and the estimate angles (right). The results are from the same time instance as reported in [7], and the reader is referred to this publication for the biases and SD of the velocity estimation method itself. For each graph the median and the interquartile range error is estimated and are denoted by the solid and the dotted red line, respectively. It can be noted that on both the moving average
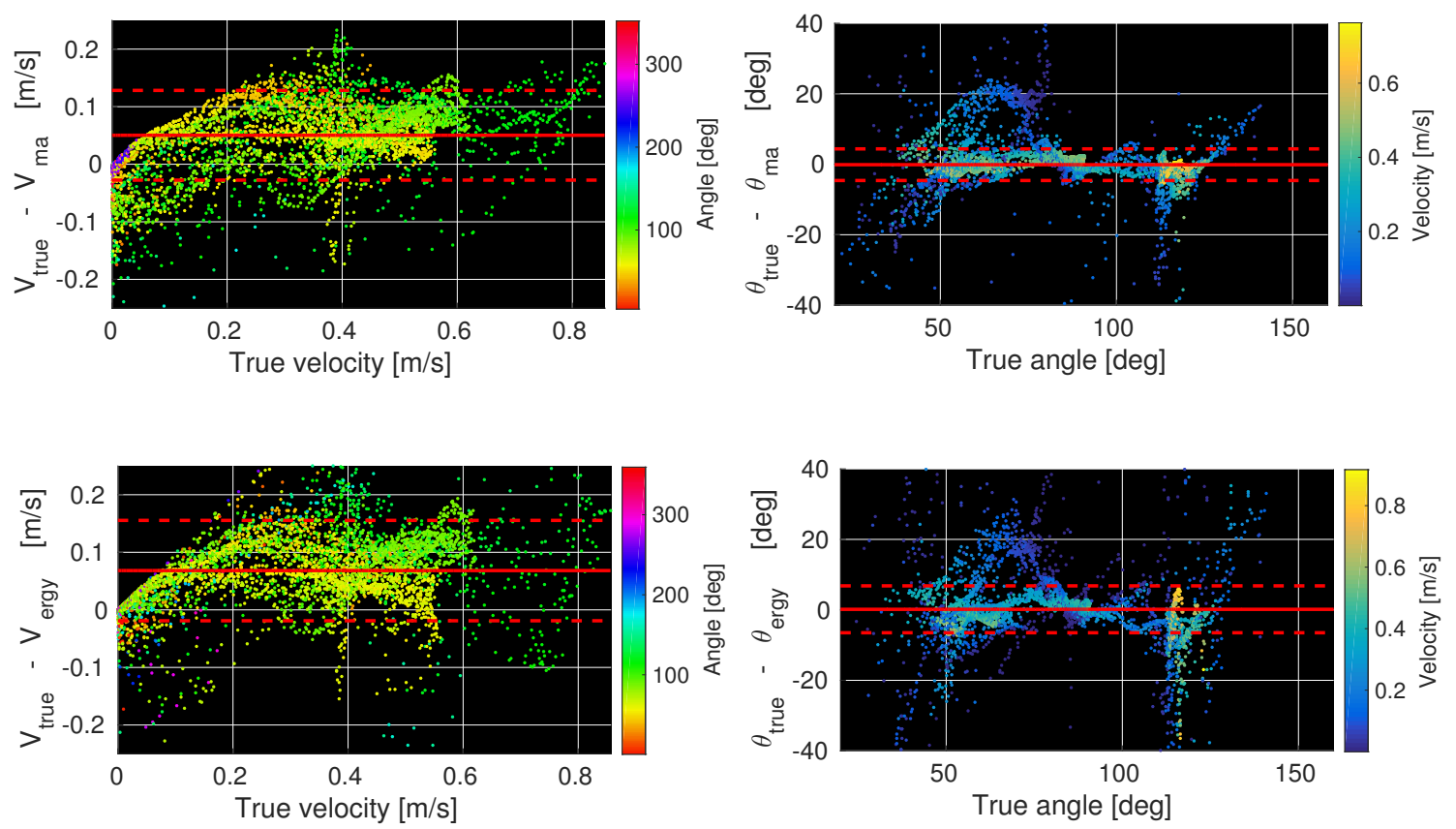

Fig. 3. Scattered error plots for the velocity magnitude (left) and angles (right) for: the moving average (top) and the energy based (bottom). The median and the interquartile ranges of the differences are denoted by the solid and the dotted red line, respectively. 
and energy based under-estimation of the velocity magnitudes are present with being slightly larger for the energy based with $-7.95 \% \pm 10.1 \%$ compared to $-5.83 \% \pm 9.08 \%$ from the moving average filter. This arises from the under estimation that occurs when angles close to $90^{\circ}$ are present. On the other had, the estimated velocity angles errors remains almost unaffected at $0.15^{\circ} \pm 6.65^{\circ}$ and $-0.12^{\circ} \pm 4.48^{\circ}$, for the energy and moving average respectively.

In vivo measurements in Fig. 4 show a significant improvement during the peak systole stage, where the movement of the vessel wall is the largest.
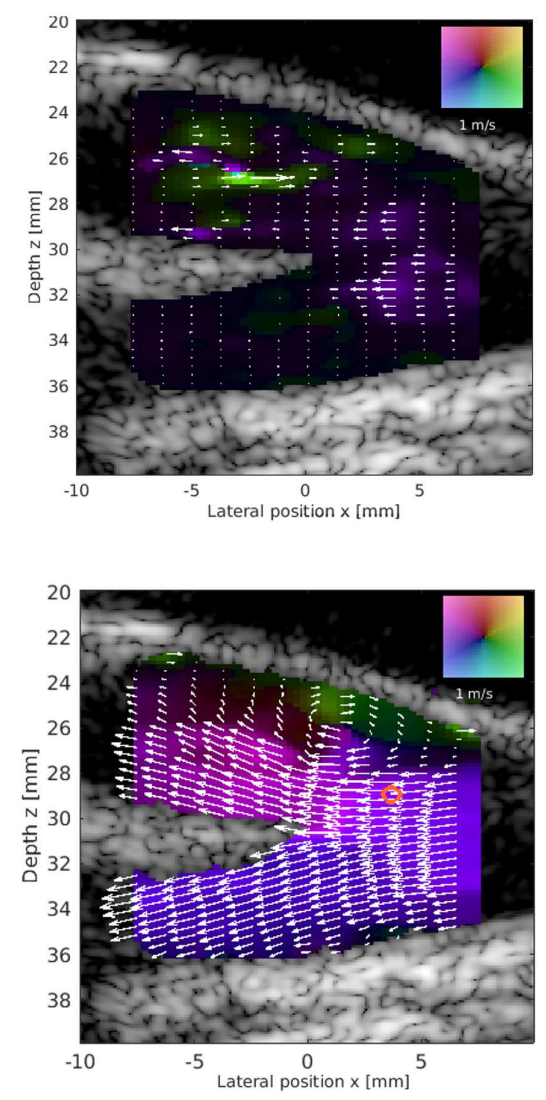

Fig. 4. Vector flow images of the carotid bifurcation of a healthy 27 yo during the peak of the vessel wall movement when using: the moving average filter (top), the energy based filter (bottom).

\section{DISCUSSION}

One of remaining questions is the threshold which, as mentioned in Section II, ideally must be selected just above the $G_{\text {blood }}$ peak value. However, this peak value would vary with the flow velocity, since it is dependent on the transit-time of the scatterers $\left(\sigma_{\text {blood }}\right)$. Furthermore, the peak value also varies both spatially and in time depending on the imaging depths, imaging angles, and individual patient differences.

Nevertheless, the approach is robust to these variations as long as the area under the curve from the tissue spectrum is not larger than the one under the blood spectrum, as shown in Fig. 1. This characteristic gives the proposed method a relatively wide operation range, allowing the use of a single threshold for imaging regions of similar imaging characteristics (e.g. carotid bifurcation) among different individuals. It is worth mentioning that for a more general use the authors have developed a method for the detection of the threshold based on an adaptive Gaussian model [10].

Another factor affecting the energy based filter performance is the spectral leakage from the tissue signal, which larger temporal sidelobes could hinder the flow signal. Therefore, windowing before the FFT is used to ameliorate this effect. It is also important to notice that longer packet lengths than the standard CFM techniques are needed $(>32)$, the reason being that the flow signal needs to be well resolved from the nearby frequencies of the tissue. Therefore, the use of high acquisition rates with continuous data is beneficial for the well functioning of the energy based filter.

\section{CONCLUSION}

A method for removing of the tissue signal for the estimation of vector flow images has been presented. The approach is based on the energy content of the signal spectrum instead of the frequencies. Simulations of the proposed approach has shown behaviour similar to a moving average filter in the absence of tissue movement. However, in the presence of tissue movement it outperforms the frequency based filter. The in vivo results also showed a significant improvement for the estimation of the flow on the carotid bifurcation region.

\section{REFERENCES}

[1] A. C. H. Yu and L. Løvstakken, "Eigen-based clutter filter design for ultrasound color flow imaging: a review," IEEE Trans. Ultrason., Ferroelec., Freq. Contr., vol. 57, no. 5, pp. 1096-1111, 2010.

[2] C. Demene, T. Deffieux, M. Pernot, B.-F. Osmanski, V. Biran, J.-L. Gennisson, L.-A. Sieu, A. Bergel, S. Franqui, J.-M. Correas, I. Cohen, O. Baud, and M. Tanter, "Spatiotemporal clutter filtering of ultrafast ultrasound data highly increases Doppler and fUltrasound sensitivity," IEEE Trans. Med. Imag., vol. 34, no. 11, pp. 2271-2285, 2015.

[3] A. C. H. Yu and R. S. C. Cobbold, "A new eigen-based clutter filter using the Hankel-SVD approach," in Proc. IEEE Ultrason. Symp., 2006, pp. 1079-1082.

[4] H. Torp, K. Kristoffersen, and B. A. J. Angelsen, "Autocorrelation techniques in color flow imaging: Signal model and statistical properties of the autocorrelation estimates," IEEE Trans. Ultrason., Ferroelec., Freq. Contr., vol. 41, pp. 604-612, 1994.

[5] L. Løvstakken, S. Bjærum, and H. Torp, "Optimal velocity estimation in ultrasound color flow imaging in presence of clutter," IEEE Trans. Ultrason., Ferroelec., Freq. Contr., vol. 54, no. 3, pp. 539-549, March 2007.

[6] J. A. Jensen, H. Holten-Lund, R. T. Nilsson, M. Hansen, U. D. Larsen, R. P. Domsten, B. G. Tomov, M. B. Stuart, S. I. Nikolov, M. J. Pihl, Y. Du, J. H. Rasmussen, and M. F. Rasmussen, "Sarus: A synthetic aperture real-time ultrasound system," IEEE Trans. Ultrason., Ferroelec., Freq. Contr., vol. 60, no. 9, pp. 1838-1852, September 2013.

[7] J. Jensen, C. A. Villagomez-Hoyos, M. B. Stuart, C. Ewertsen, M. B Nielsen, and J. A. Jensen, "Fast plane wave 2-D vector flow imaging using transverse oscillation and directional beamforming," IEEE Trans. Ultrason., Ferroelec., Freq. Contr., vol. 64, no. 7, pp. 1050-1062, 2017.

[8] J. A. Jensen, "A multi-threaded version of Field II," in Proc. IEEE Ultrason. Symp. IEEE, 2014, pp. 2229-2232.

[9] A. Swillens, L. Løvstakken, J. Kips, H. Torp, and P. Segers, "Ultrasound simulation of complex flow velocity fields based on computational fluid dynamics," IEEE Trans. Ultrason., Ferroelec., Freq. Contr., vol. 56, no. 3, pp. 546-556, 2009.

[10] C. A. Villagomez-Hoyos, "Synthetic aperture vector flow imaging," $\mathrm{Ph} . \mathrm{D}$. dissertation, Technical University of Denmark, 2016. [Online]. Available: http://findit.dtu.dk/en/catalog/2347162876 